\title{
Workforce-efficient Consensus in Crowdsourced Transcription of Biocollections Information
}

\author{
Andréa Matsunaga ${ }^{\mathrm{a},{ }^{*}}$, Austin Mast ${ }^{\mathrm{b}}$, José A. B. Fortes ${ }^{\mathrm{a}}$ \\ ${ }^{a}$ Advanced Computing and Information Systems Laboratory, ECE, University of Florida, 32611, USA \\ ${ }^{a}$ Department of Biological Science, Florida State University, 32306, USA
}

\begin{abstract}
Crowdsourcing can be a cost-effective method for tackling the problem of digitizing historical biocollections data, and a number of crowdsourcing platforms have been developed to facilitate interaction with the public and to design simple "Human Intelligence Tasks". However, the problem of reaching consensus on the response of the crowd is still challenging for tasks for which a simple majority vote is inadequate. This paper (a) describes the challenges faced when trying to reach consensus on data transcribed by different workers, (b) offers consensus algorithms for textual data, (c) implements a consensus-based controller to assign a dynamic number of workers per task and per field of a task, (d) proposes the use of clustering to further eliminate redundant work and (e) proposes enhancements of future crowdsourcing task assignments in order to minimize the need for complex consensus algorithms. Experiments using the proposed algorithms show multifold increase in the ability to reach consensus when compared to majority voting using exact string matching. In addition, the workforce controller is able to decrease the crowdsourcing cost per task and per task field by $37 \%$ and 50\%, respectively, when compared to a strategy that uses a fixed number of workers. The accuracy of clustering is also good and it has the potential to increase the quality of tasks that can be clustered.
\end{abstract}

Keywords: crowdsourcing; consensus algorithms; digitization; biocollections infrastructure; biodiversity

\section{Introduction}

Digitization of the scientific information associated with specimens is at full speed in U.S. biological research collections, as part of the NSF-ADBC (Advancing Digitization of Biodiversity Collections) program [1]. The materials requiring transcription include catalogues, field notes, and especially specimen labels (e.g., labels on specimen sheets or packets, slides, drawers, pins, vials and jars). Fig. 1 shows a sample of the material to be digitized and exposes some of the challenges faced when trying to apply Optical Character Recognition (OCR): handwriting, different types of fonts, faded text, inconsistent positioning of labels, imaging at oblique angles, specimens covering and sometimes hiding text, and the need to interpret or infer information. Crowdsourcing is an appealing solution to lower the cost and accelerate the transcription rate of the large volume of information in these labels which have been amassed over the last two centuries [2]. Several projects [3][4][5][6][7] are proving that volunteers from the general public can perform the transcriptions at speeds that a single project or institution cannot match.

The strategies to setup such a crowdsourcing platform vary, but all of them need to deal with the fact that workers are not always reliable. Given the importance of transcription accuracy for scientific studies, crowdsourced transcriptions require some amount of redundancy. At the same time, there is a trade-off between the level of redundancy (multiple workers transcribing the same material) and the total number of specimen labels transcribed due to limits on available volunteer hours or budgets (when transcribers are compensated). Furthermore, when multiple independent workers transcribe the same material, it is necessary to generate a final consensus amongst all redundant transcriptions. Reaching consensus on a numeric value or on a fixed number of categories is straightforward with majority voting, but textual transcriptions require further effort, especially in the presence of human interpretation of the information contained in specific label fields (leading to non-verbatim transcriptions).

To deal with these challenges in crowdsourcing systems, this paper describes (1) the influence of string comparison algorithms on reaching consensus using transcribed and interpreted data from a popular crowdsourcing platform for digitization of biocollections (NotesFromNature [3]), (2) a strategy to produce a consensus response with excellent accuracy, (3) a controller that minimizes the number of workers required for a particular task and for each field of a task, (4) use of clustering to further minimize the workforce requirement and improve the quality of certain tasks, and (5) insights to improve the design of crowdsourcing tasks.

\footnotetext{
* Corresponding author. Tel.: +1-352-392-4964; fax: +1-352-392-5040.

E-mail address: ammatsun@ufl.edu.
} 
This paper is organized as follows: Section 2 presents related work, Section 3 details our proposed consensus algorithms and worker controller, Section 4 shows our experimental results, Section 5 presents improvements for future work, and Section 6 concludes this work.

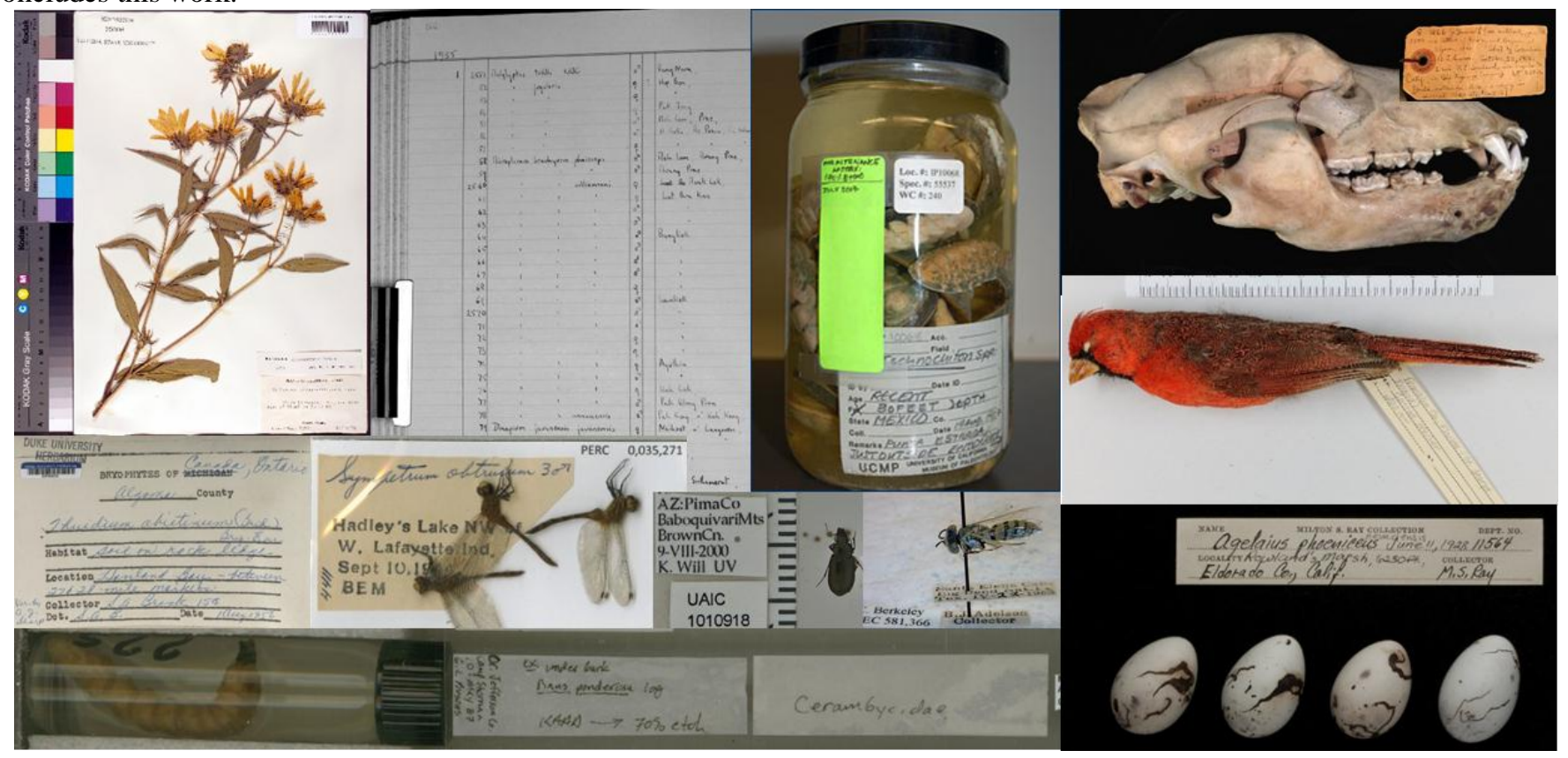

Fig. 1. Materials to be digitized: catalogues (center top), and labels next to specimens. Labels appear in jars (top center right), sheets of paper with pressed specimens (top left), vials (bottom left), packets (center left), pinned (center right), hanging from specimens (top right) and in drawers (bottom right).

\section{Related Work}

Several projects and software tools have started to address the particular scenario of biological collection transcription. Some take advantage of OCR to generate as much transcription as possible, and Natural Language Processing (NLP) to parse information from OCR into appropriate fields. Noteworthy efforts are shown in Table 1. The workflow strategies of these projects vary greatly: NotesFromNature [3] requires a fixed number of transcriptions to consider a specimen digitization task complete; Atlas of Living Australia's DigVol (ALA DigiVol) [4] and Symbiota [5] require a single transcription; ScioTR [6] produces alternatives from OCR and NLP, from which the user selects the best option; and Transcribe Bentham [7][8] makes use of multiple collaborative volunteers. The need for consensus is either avoided (where there are single or collaborative transcribers), or is turned into another crowdsourcing task, or is simply absent with multiple answers returned to the task requestor. General purpose crowdsourcing platforms, such as Amazon Mechanical Turk [9] and PyBossa [10], have enabled a number of studies where the tasks required only a simple majority voting while surveying users [11], categorizing content [12][13], assessing translation [14][15], among numerous other micro-tasks. Enki [16] is an analysis tool for generating simple statistics (e.g., counts, unique values, and averages) on crowdsourcing tasks produced through PyBossa, and it does not include consensus algorithms for textual data. Thus, our contributions complement all these crowdsourcing platforms by increasing the confidence in accuracy with use of multiple workers and offering consensus on multiple answers.

Table 1. Crowdsourcing Transcription Projects

\begin{tabular}{|c|c|c|}
\hline Platform & Platform & Strategy \\
\hline NotesFromNature [3] & Zooniverse & $\begin{array}{l}\text { Fixed number of workers, no consensus mechanism, custom } \\
\text { interfaces per label type (e.g., plants, fungus, or insects). }\end{array}$ \\
\hline ALA DigiVol [4] & Grails & $\begin{array}{l}\text { Single worker followed by expert approval, and custom fine- } \\
\text { grained fields. }\end{array}$ \\
\hline Symbiota [5] & PHP & $\begin{array}{l}\text { Single worker, ability to load data from OCR and NLP, fine- } \\
\text { grained fields. }\end{array}$ \\
\hline ScioTR [6] & Windows 8 app & $\begin{array}{l}\text { Integrates OCR and NLP, with app to select image regions, } \\
\text { sending cases where OCR/NLP fails to crowdsourcing. }\end{array}$ \\
\hline $\begin{array}{l}\text { Transcribe Bentham } \\
{[7][8]}\end{array}$ & MediaWiki & $\begin{array}{l}\text { Multiple collaborative workers, with moderation by experts. } \\
\text { Transcription of verbatim text from a page. }\end{array}$ \\
\hline
\end{tabular}


Data quality on crowdsourced tasks has been widely studied by analyzing how human factors influence responses [12], offering methods to separate error from bias [13], comparing responses from the crowd with those provided by experts [14][15][17], detecting and filtering noisy workers based on their z-score [18] or a support-vector-machine algorithm [19], analyzing the use of a small amount of expert data to supervise the crowd responses [20], and proofing the response of workers with another crowdsourcing task [21]. In this work, we also assess the quality of the consensus output by comparing with transcriptions performed by an expert.

Minimizing the amount of crowdsourcing work, and consequently its cost, has mainly been tackled by the addition of a phase to validate the responses from previous volunteers by experts [4][5][7] or other workers [19][21]. However, this puts pressure on experts and/or more weight on the quality of workers validating the data. In this work, we propose to control the number of workers on a per-task basis, based on the consensus reached by responses from independent workers. The main idea behind the controller is that the number of transcriptions needed to reach consensus will depend on the difficulty and amount of interpretation required by the task and its sub-parts, while providing a threshold level of accuracy. TurKit [22] is a general purpose toolkit for interactive programming against Amazon Mechanical Turk platform, and it can be leveraged to integrate our controller into a widely used platform. The work in [23] proposed minimization of the crowdsourcing cost while maintaining a certain level of reliability based on the inferred reliability of a worker. Unlike [23], which finds a fixed optimal number of tasks given the level of reliability of the worker population, our controller dynamically changes the number of workers, recognizing the heterogeneity in task difficulty. We also prefer a method that does not depend on grading workers on the basis of their past performance, because their performance can vary over time and most volunteering portals do not require workers to sign up in order to lower their entrance barriers. As a consequence, we cannot weight a substantial amount of transcriptions. For example, Fig. 2 shows that $20 \%$ of crowdsourcing transcriptions evaluated in this paper come from anonymous volunteers.

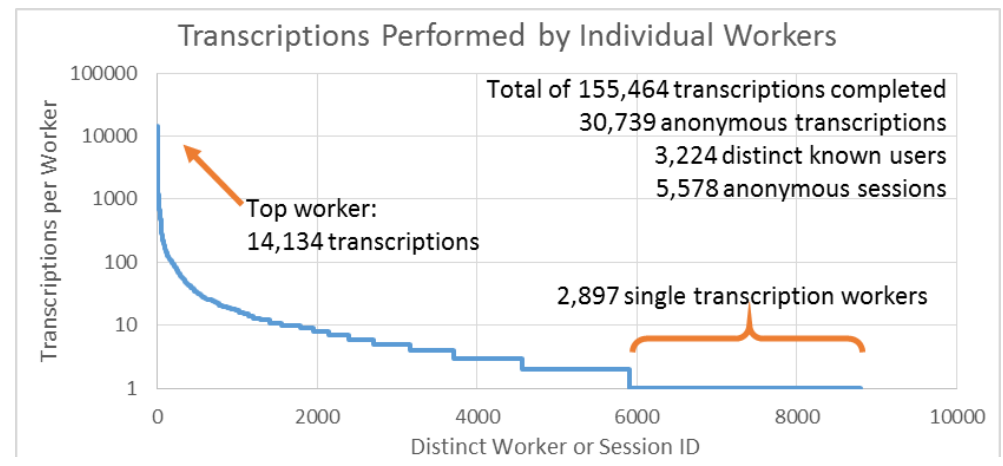

Fig. 2. Long-tail distribution of transcriptions completed per worker: a few workers are responsible for completing the same number of transcriptions as those of many workers completing a few. The number of anonymous transcriptions is substantial (30,739 out of 155,464 total transcriptions).

\section{Consensus and Worker Controller}

Before detailing the proposed consensus algorithms, it is worth describing the type of transcription considered for crowdsourcing and emphasizing the requirement for interpretation that leads to responses that are challenging to score. Fig. 3 shows a typical image of a herbarium specimen containing the label (enlarged) that needs to be transcribed. For this particular task, the worker is asked to transcribe the information into 10 separate fields: (1) country, (2) state, (3) county, and (4) descriptive location (including elevation and georeference) where the specimen was collected; (5) scientific name and (6) authorship of the name; (7) specimen's habitat and description (habitat henceforth), (8) name of one or more collectors; (9) a number assigned to the specimen by the collector; and (10) the date when the specimen was collected. Different colors are used to indicate the level of difficulty observed for each field. Very specific fields (e.g., state and date) with a limited number of options are easy (shown in green) to transcribe. Fields in yellow are more challenging due to the possibility of mistakes in parsing the information. For example, not all volunteers are familiar with the nomenclatural practice of including the author of the scientific name on the label; this is especially complicated when the name is at the sub-specific rank since the authorship information is interleaved with other components of the name. Some specimens also have multiple determinations (i.e., scientific name identifications). While only the last determination is expected, different workers might transcribe earlier names or all names. In addition, if one is not used to the layout of these labels, it is possible to confuse the determiner's name with the collector's name. The hardest fields (in red) are habitat and location due to the typical interleaving of this information with other content on the label, the occasional need to rephrase the information in the label, and the length of this information. It is also difficult to compare and grade the work, when distinct workers might invert the order of sentences, use abbreviations or expand them, make mistakes in different regions of the text, especially in scientifically relevant and numeric data, possibly include redundant information (e.g., repeat county information in location), be explicit about absence of information and apply alternative approaches to punctuation and character encoding (e.g., Löve vs. Love). 


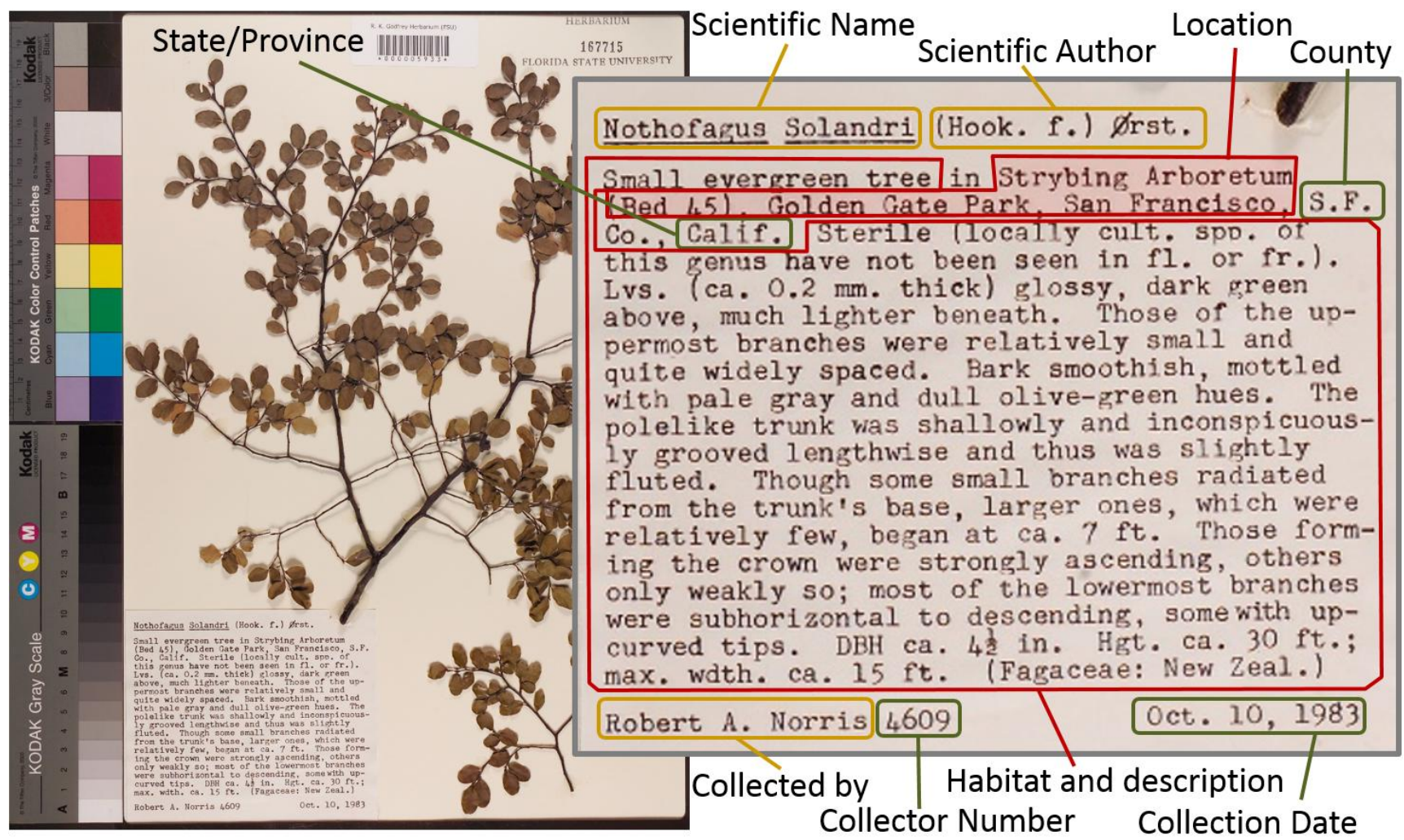

Fig. 3. Typical crowdsourcing task. A herbarium sheet requiring transcription is shown on the left. Its label is enlarged (on the right), showing the expected values for 9 fields to be transcribed: state/province, county, location, scientific name, scientific name author, habitat and description, collector, collector number, and collection date. An additional country field needs to be inferred by the user from the state information. Colors of rectangles enclosing text indicate level of difficulty in transcription (green indicates easiest, red indicates hardest, and yellow indicates in-between transcription difficulty).

To deal with all these challenges present in textual data comparison in the process of reaching consensus, and to minimize the number of workers and the work required for each task, we propose the workflow depicted in Fig. 4. The workflow uses lossless algorithms to normalize the answers from different workers and produce uniform output, and lossy algorithms to accept the equivalency of two different answers and err on the side of the most complete response. The minimum number of workers is controlled on the basis of the ability of distinct workers to reach consensus. In the case of per-field consensus, the controller queues tasks with specific fields to be transcribed (not including fields that have reached consensus), and information on fields that reached consensus are stored together with past transcriptions for the task. Consensus is based on majority voting and can make use of approximate matching offered by lossy algorithms. In addition, explicit normalization of responses indicating absence of information is always performed as part of the lossless algorithm execution. Details of all algorithm options, as well as an alternative voting strategy, are shown in Table 2 .

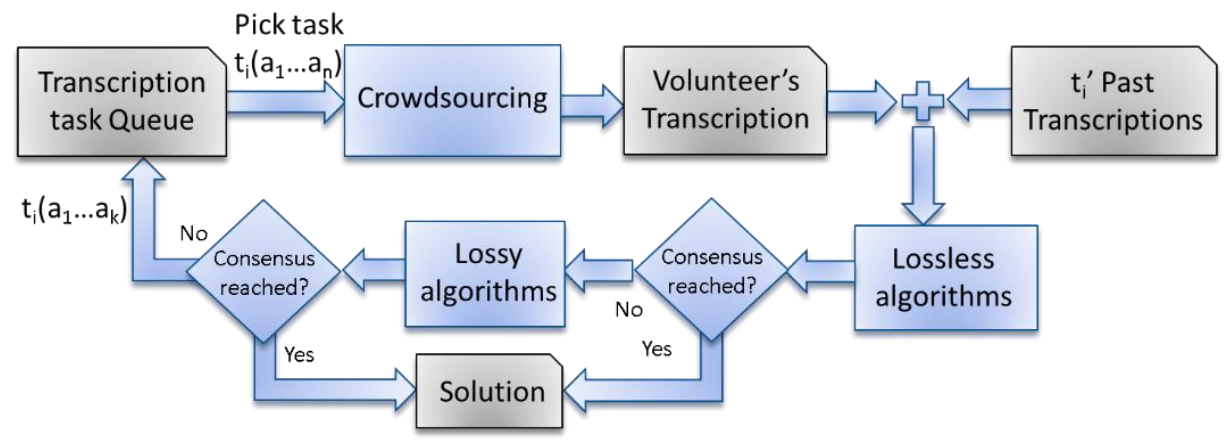

Fig. 4. High-level view of proposed solution for reaching consensus with the minimum number of transcriptions, by controlling the amount of workers and when to apply lossless and/or lossy algorithms for a specific task. 
Table 2. Lossy and Lossless Algorithms

\section{Lossless normalization algorithms:}

b Removes all extra whitespaces.

$\mathrm{n}$ Apply specific transformation functions on a per-field basis (e.g., to normalize section/township/range, proper names, and latitude/longitude).

$\mathrm{t}$ Apply specific translation tables on a per-field basis (to expand abbreviations, e.g., hy, hwy, and hiway to highway) or to shorten expansions (e.g., Florida to FL).

\section{Lossy normalization algorithms:}

w Approximate comparison by ignoring all whitespace (e.g., " $0-3$ " is equivalent to " $0-3$ ").

c Case insensitive approximate comparison (e.g., "Road" and "road" are considered equivalent).

s Consider two sequences equivalent when one is a substring of another or one sequence contains all words from the other.

p Punctuation insensitive approximate comparison (e.g., separation of sentences with comma, semi-colon or period are considered equivalent).

f Approximate fingerprint comparison ignoring the order of words in sentences.

1 Approximate equivalency when sequences have Levenshtein distance [24] within a configurable threshold (12 indicates a maximum distance of 2).

\section{Alternative voting and consensus output:}

e Considers minimum number of workers and voting on a per field basis.

$\mathrm{v}$ Consensus is reached when there is a single group (set of matching answers) that has the most votes, instead of requiring strict majority vote among all answers.

a Outputs best available answers when consensus is not achieved.

\section{Experimental Setup and Results}

The experimental data evaluated in this work was obtained from NotesFromNature [3] for an application transcribing herbarium specimens (exemplified in Fig. 3) from three institutions: Florida State University (FSU), University of South Alabama (USAM) and Valdosta State University (VSC). It consists of a set of 155,464 total transcriptions completed by 8,802 distinct workers or sessions (Fig. 2) for 40,371 distinct tasks. The FSU tasks were initially configured to require 10 workers per task, and later modified to require 4 workers per task, while all USAM and VSC tasks were configured to require 4 workers. As shown in Fig. 5, the distribution of workers per task is different for each institution and each task received a different number of workers after removing fully void responses, responses that took no time to complete and skipped tasks. Note that consensus cannot be reached for tasks assigned to a single worker (thus, maximum consensus achievable in these experiments is $95.64 \%$ ). The variance in responses and the level of difficulty in each field can be shown by the number of unique distinct values per field summarized in Table 3. Location and habitat have, respectively, an average of 2.7 and 2 distinct values per crowdsourcing task (i.e., per specimen label). The amount of unique values for "collected by" can also be considered high given that the variation is expected to be small when all specimens in this dataset came from a few institutions, indicating the need for normalization algorithms.
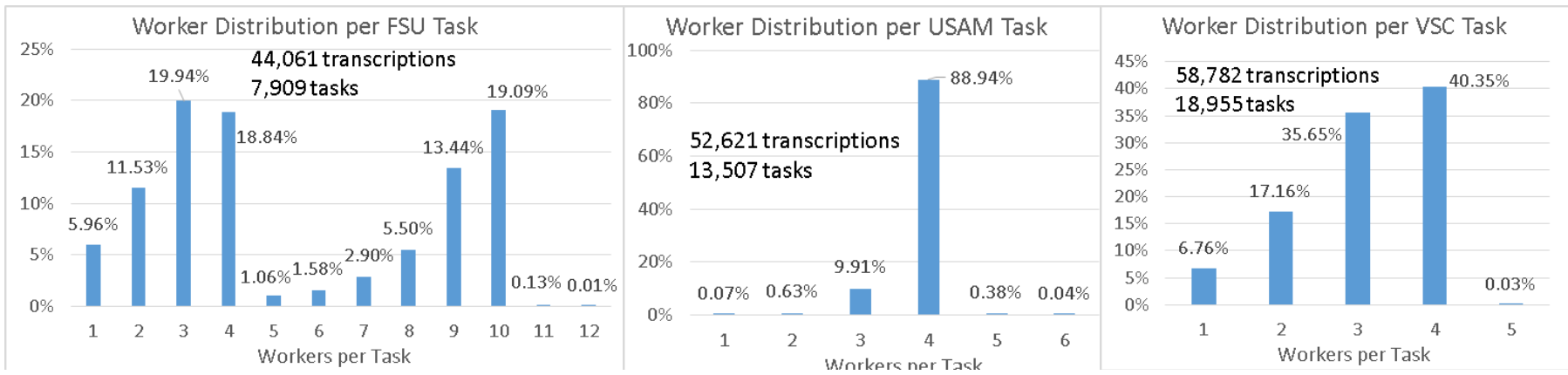

Fig. 5. Distribution of workers per task on the experimental dataset from NotesFromNature on the transcription of herbarium specimens from Florida State University (FSU), University of South Alabama (USAM) and Valdosta State University (VSC).

Table 3. Distribution of Unique Values Per Field for 40,371 Distinct Tasks

\begin{tabular}{llllllll}
\hline Field & Uniq\# & Field & Uniq\# & Field & Uniq\# & Field & Uniq\# \\
\hline Country & 93 & Location & 107,997 & Scientific name & 26,461 & Collector number & 25,502 \\
State/Province & 813 & Habitat & 81,029 & Scientific author & 4,032 & Collection date & 10,044 \\
County & 1,861 & Collected by & 15,847 & & & & \\
\hline
\end{tabular}




\subsection{Consensus Achievement}

To evaluate the influence of different combinations of lossless and lossy algorithms, a set of configurations using an increasing number of algorithms was defined. Configurations are labeled with the combination of single letter options from Table 2, with 'nf' indicating no use of lossless/lossy functions. Fig. 6 shows the percentage of tasks reaching consensus in 6 distinct configurations, on all 10 transcribed fields (complete consensus series), as well as consensus achievement per field. It confirms that location and habitat are the most challenging fields for reaching consensus, and that country, state, county, collector number, and collection date can reach consensus at a high rate without the added textual data comparison algorithms. While country displays consensus achievement of $95.1 \%$ with exact matches, location achieved only $11.2 \%$ consensus. This is due to the fact that fields with high consensus achievement with exact matches offer a multiple choice drop-down in the user interface, minimizing the amount of variation between responses (e.g., "U.S.A.", vs. "USA", vs. "United States" in the country field) and minor misspellings.

"Collected by" had considerable improvement (from 66.3\% to 81.3\%) only with the use of lossless algorithms (bnt case) due to the custom normalization of proper names. This normalization function ensures proper capitalization of names (only the first letter), use of punctuation (abbreviations have periods), and use of special symbols as " $\&$ " as shown in Table 4.

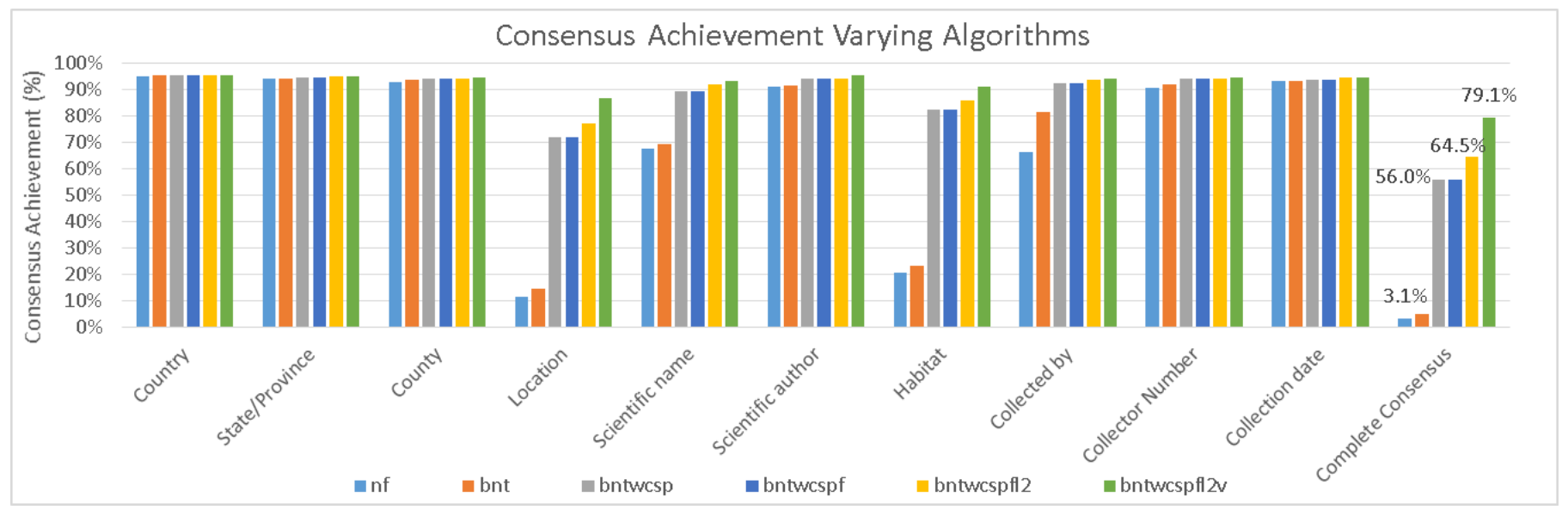

Fig. 6. Consensus achievement varying the lossless and lossy algorithms applied to crowdsourced data (each distinct series represents a set of algorithms; abbreviations are as in Table 2). Consensus achievement for each of the 10 transcribed fields and overall consensus of all fields (complete consensus) show that consensus achievement is improved as more algorithms are added, with a 25 -fold improvement when applying all algorithms over the no normalization case.

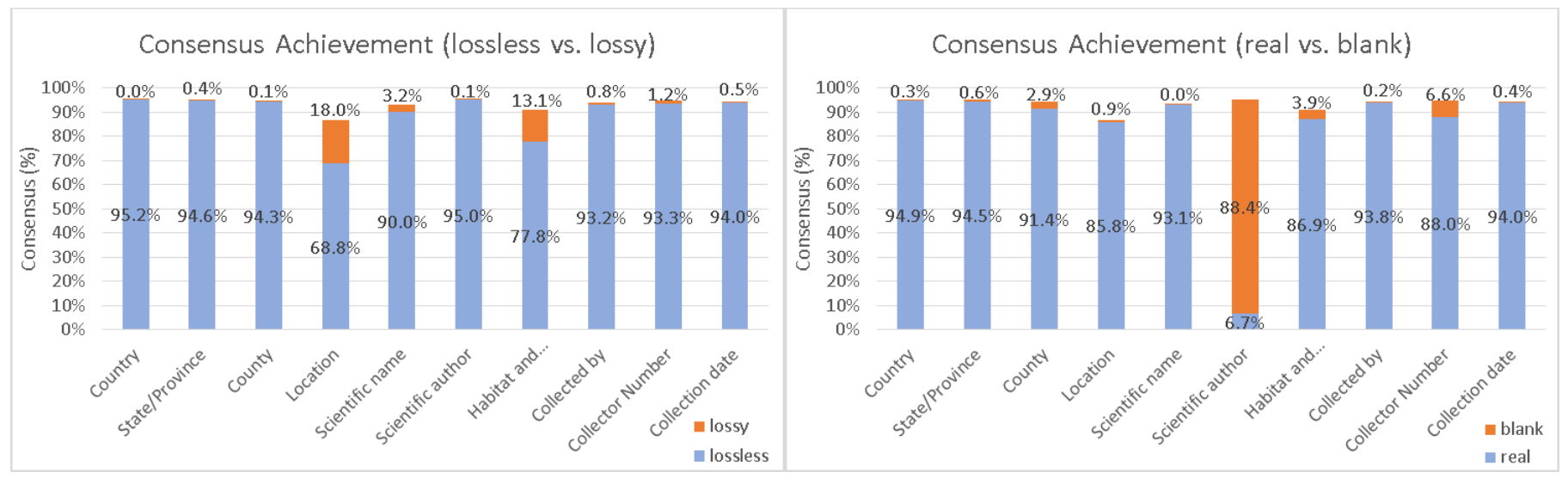

Fig. 7. Percentage of crowdsourcing tasks achieving consensus after applying lossless or lossy algorithms (on the left), and percentage of responses resulting in consensus with either blank or non-blank output (on the right, with blank indicating absence of information in the transcription task).

In all cases, incremental use of lossless and lossy algorithms improved the ability to reach consensus, with the complete consensus being improved from $3.1 \%$ to $79.1 \%$ (nf vs. bntwcspfl2v). The use of Levenshtein distance has considerable impact on location and habitat fields (18\% and 13.1\% of tasks respectively in Fig. 7 using lossy algorithms) due to verbosity of these fields 
and potential for minor differences in transcription, leading to $8.5 \%$ improvement in overall consensus. Fig. 7 (right) also shows that all fields present good amount of completeness (non-blank responses), except for scientific author, which was removed from the task interface after the launch of the system. Since lossy algorithms only group similar answers, the voting strategy accepting the group with most votes (bntwcspfl2 vs. bntwcspfl2v, i.e., use of option v) has also a substantial impact on the complete consensus (14.6\%). To exemplify the impact of voting, consider the non-normalized data in Table 4 and assume that all additional workers provide the desirable answer "Richard W. Pohl". The strict majority voting strategy on non-normalized values would require 4 additional workers, while the voting scheme considering the group with most votes would require only one additional worker.

Table 4. Example of Proper Name Normalization

\begin{tabular}{llll}
\hline Collected by & Count & Normalized & Count \\
\hline Richard W. Pohl & 2 & Richard W. Pohl & 5 \\
Richard W.Pohl & 2 & R. W. Pohl & 1 \\
Richard W Pohl & 1 & unknown & 1 \\
R W Pohl & 1 & & \\
unknown & 1 & & \\
\hline
\end{tabular}

\subsection{Accuracy of Consensus Result}

Improvements to consensus-building algorithms are essential to generate a single response for each transcription task, and are equally important for ensuring the quality of consensus output. In this subsection, we evaluate 300 tasks (out of the 40,371) for which accurate expert transcriptions are also available for comparison against consensus output. It is important to note that the expert had access to all specimen images while any transcription volunteer had access to one specimen image at a time. Since some specimens (distinct tasks) share information, the expert was able to deduce some of the information that was illegible or simply absent. The latter case happens because different parts of the same specimen are often placed on different sheets and not all sheets belonging to a single specimen receive the same label.

Accuracy was calculated based on string matching ratio $(2 *$ matches / sum(len(s1), len(s2))), where matches are the number of matching characters between the strings s1 and s2, which are the consensus and the expert entries, respectively. One exception to this calculation is when one of the strings being compared is 'unknown'. In this case, the value is either 1 if both responses are 'unknown', or 0 if only one is 'unknown'.

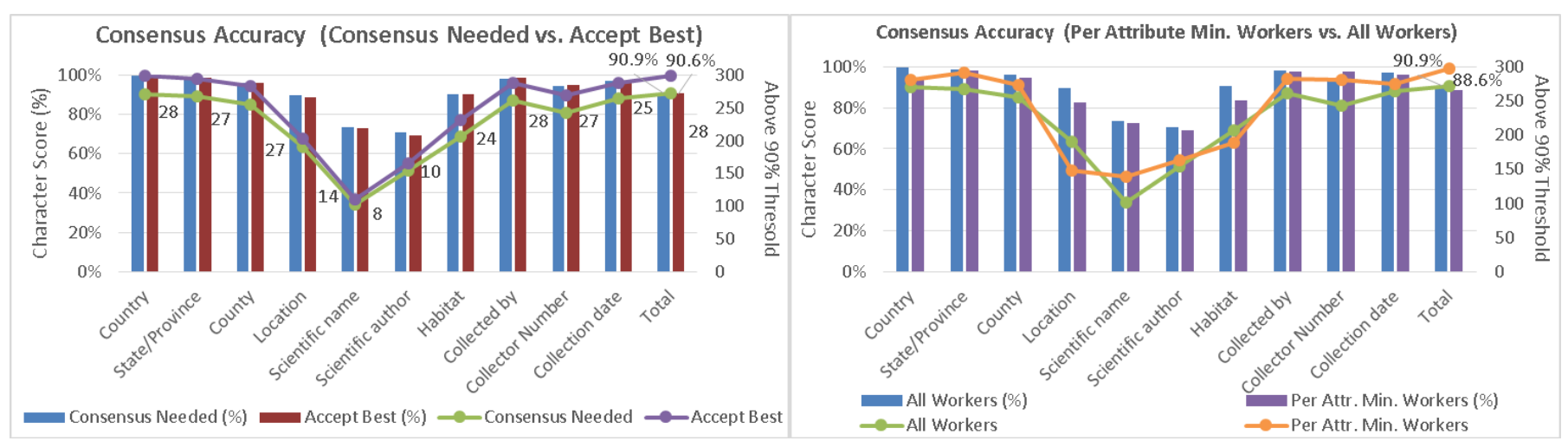

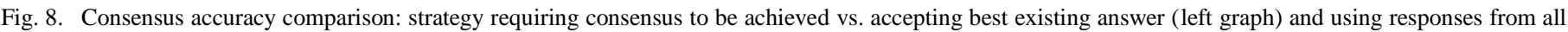
workers vs. controlling the number of workers per field in a task (right). The bars show the accuracy measured as the percentage of character matches (left axis), and the lines represent number of entries considered accurate due to at least $90 \%$ of character matches (right axis). The numbers next to the lines represent the difference between the two lines. The graphs show little impact on accuracy when controlling the number of workers to be minimal and when accepting the best response.

Fig. 8 compares the accuracy of consensus output to answer the following two questions. Can responses from workers be accepted even if there is no consensus among the responses? How much is the accuracy affected by using the minimum number of workers per task and per field of a task established by the controller? The answer to both questions is that the effect on the overall accuracy is minimal: $0.3 \%$ drop for accepting cases that did not reach consensus and $2.3 \%$ for minimizing the needed workforce on a per task field basis (the left-most bars or 'Total' in the graphs). The graph showing the impact minimizing workers per task has been omitted since results were similar to minimizing workers per task field (right graph), with a decrease in accuracy of only $1.6 \%$. These graphs also show the number of accurate responses (lines in the graph), i.e., the number of responses with $90 \%$ or more of characters matching the expert data. In the left graph, the number of accurate responses when requiring consensus to be 
achieved is lower than when the best answer is accepted without consensus because only 272 out of 300 evaluated tasks reach consensus. Thus, it is interesting to evaluate the difference between the two lines: country can be accurate in all 28 cases where consensus is not reached, but only 8 are acceptable for scientific name (a field that requires accuracy for future scientific exploration of this biodiversity data). The right graph shows a surprising result - when reducing the number of workers, a greater number of tasks can reach consensus (297 vs. 261), which leads to a greater number of acceptable responses that are nearly accurate. The inversion of this trend occurs only for location and habitat, because the consensus algorithms tend to prefer longer responses, and worker minimization tends to lower the chances of having a thorough worker that will transcribe all details. The inverse effect can be observed for scientific name, where workers tend to erroneously include the author, creating a longer version that is preferred by the consensus algorithms. With fewer workers, the chance of not getting names including author is higher, leading to more accurate consensus (without author).

\subsection{Workforce Savings}

Since we have demonstrated the accuracy of controlling the number of workers through consensus achievement, in this subsection we show that workforce savings can be as high as 32.96\% for the distribution in the studied dataset (Fig. 9 left). In fact, this graph shows that if a similar system is designed to use a fixed number of workers, 3 workers appear to be a good fixed setting. This result is consistent to previous work showing that a task does not need a high number of replications to achieve an acceptable level of accuracy [13]. The advantage of our controller is that 3 workers is just a good average, and our results show that there are cases where overall consensus was not reached even with 10 workers. As indicated by [12][23], the suitable number of workers also depends on the crowd that is attracted to the task and the reliability of workers. We believe that our controller is capable of adjusting to these changes without requiring extra effort from the requester in re-submitting the task for additional crowdsourcing. Assuming each field requires equal effort from the worker, Fig. 9 (right) shows that an additional $13 \%$ of workforce savings is possible by controlling the amount of fields to be present in a task when compared to only controlling the number of workers per task. This graph also confirms that most fields (e.g., country, state, and collection date) require only 2 workers for the majority of tasks, while location and habitat are the fields requiring more workers (thus, the workforce savings).

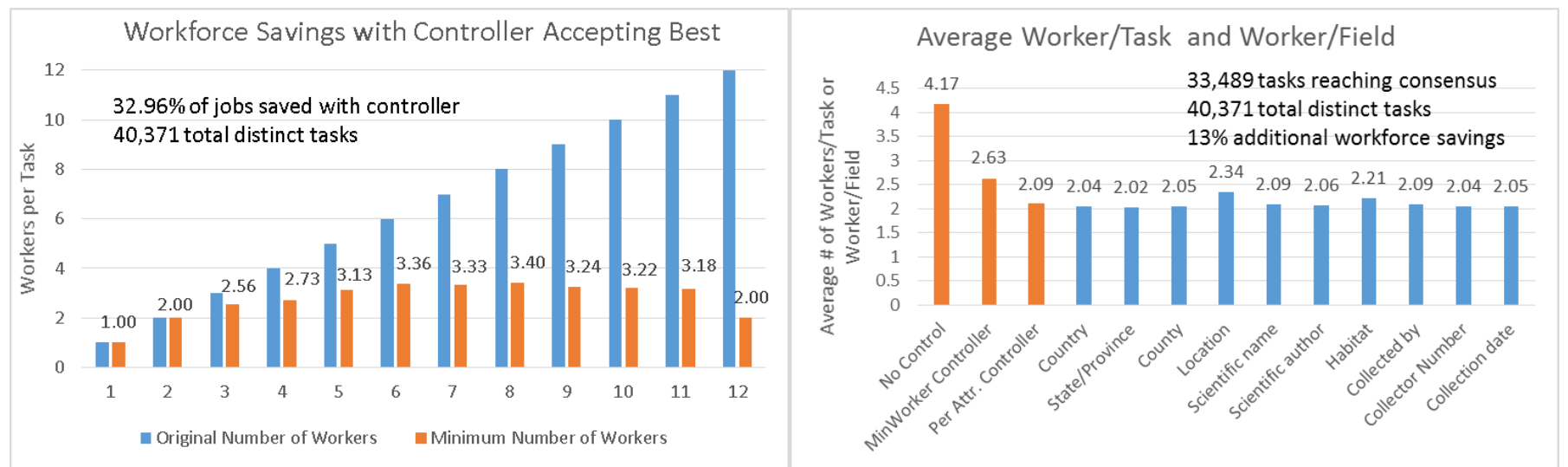

Fig. 9. Workforce savings (32.96\%) when controlling dynamically the number of workers required for a specific task based on consensus of all fields (left), and additional workforce savings of $13 \%$ (average reduction from 2.63 to 2.09 workers per task) when controlling the number of workers required per field of a task (right).

\subsection{Workforce Evaluation and Training}

While in section 4.2, accuracy of consensus response was evaluated against an expert response, obviously we cannot expect a golden dataset to exist. In this section, we evaluate the quality of work of our workforce against the consensus result and the effort required to perform transcriptions. The goal of this evaluation is not to grade our workers as some crowdsourcing platforms do, but to (a) detect at early stages workers who could benefit from training before performing additional transcriptions, (b) detect cases where it would be best to require additional workers to increase accuracy and confidence on the consensus response, and (c) detect system errors. Each transcription for a task that reached consensus was graded with the same character score described in section 4.2. These scores were averaged per worker and per task and plotted in Fig. 10, showing that: workers with good score tends to take more time to complete the task with an average of 9.18 minutes for transcriptions scoring above 0.7 (top right); a minority of 142 workers take a long time to complete a task and a minority of 165 workers complete more than 100 transcriptions (top left); a majority of 7,913 workers score above 0.7 (bottom left); and only 89 tasks scored below 0.7 , being candidates to receive additional workers (bottom right). Among the 609 workers with score below 0.7, only 202 workers completed 2 or more transcriptions and would be candidates to receive additional training. Manual inspection of transcriptions from these workers indicate that 
transcription mistakes include, but are not limited to: considerable amount of blank fields, transcription of the barcode as collector number, transcription of taxonomy determiner as collector, transcription of collector as scientific author, transcription of state as country, transcription of herbarium as location, input of bogus values, creativity in variations to indicate absence of data, use of all upper case characters, conversion of numbers into words, and repetition of the same set of values for distinct unrelated tasks.

Offering additional training addresses all these issues, but the last case, where the issue appears to be created by the crowdsourcing platform, indicates that statistics shown in this section are also important to increase reliability of the system.

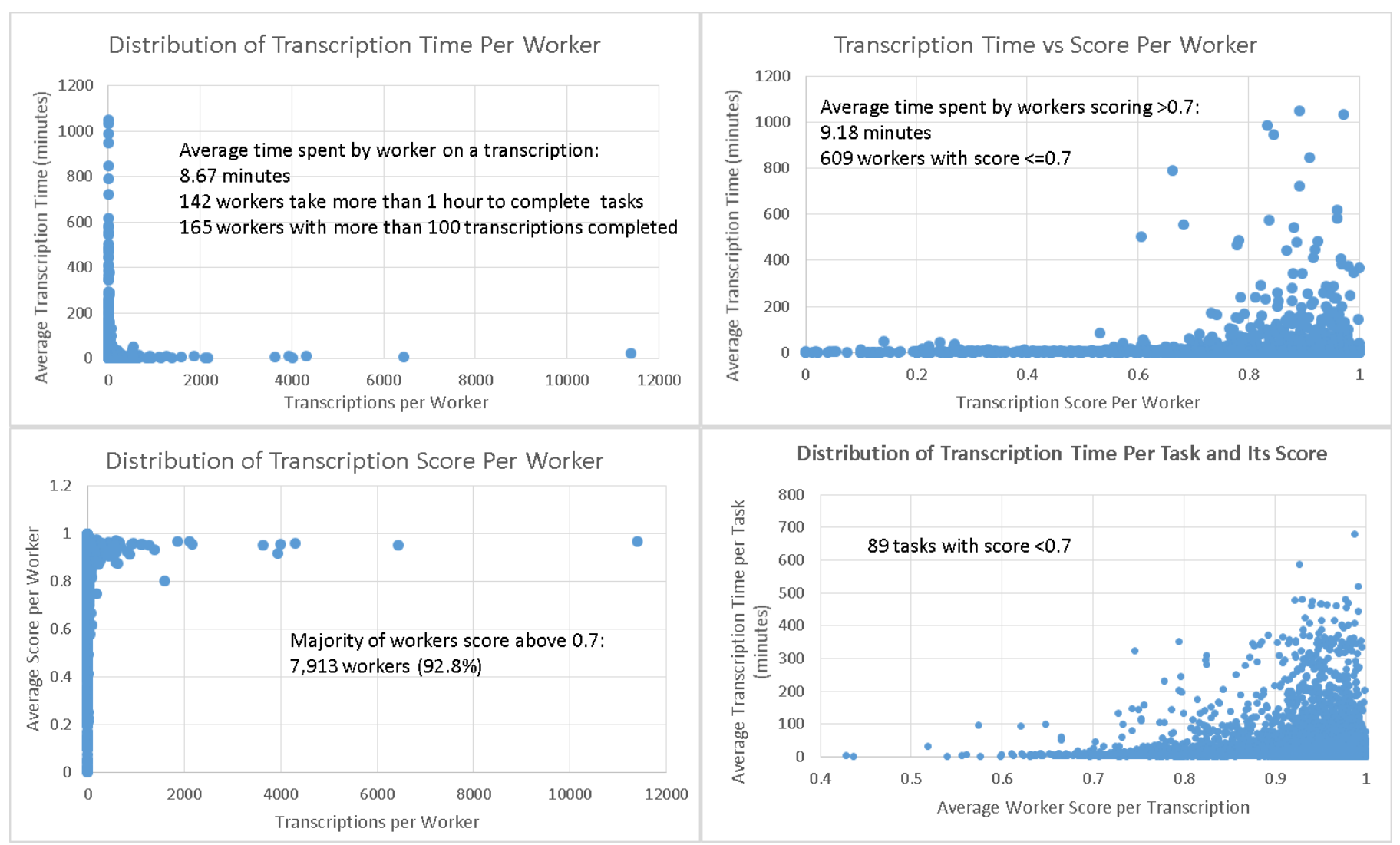

Fig. 10. Distributions of: average transcription time per worker vs. number of transcriptions per worker (top left), average transcription time per worker vs. worker score (top right), worker score vs. number of transcriptions per worker (bottom left), and average transcription time per task vs. task score (bottom right).

\subsection{Consensus Clustering}

As indicated earlier, an expert had access to all images of labels to be transcribed and could infer information that was not present in all labels. In this section, we make use of clustering techniques (a) to investigate the possibility of having an additional pass for all the tasks in a cluster to be consistent with each other, and (b) to check the possibility of decreasing even further the number of workers required for a task (i.e., a single worker is needed when a transcription belongs to a cluster of previous tasks that reached consensus). The distance between tasks that reached consensus is calculated with the same character score described in section 4.2, and clusters are formed according to an established threshold that indicates if two or more tasks belong to the same cluster. We performed clustering experiments with 300 tasks from the FSU dataset, varying the threshold, and obtained the number of clusters presented in Table 5. A graphical representation of formed clusters for 0.8, 0.85 and 0.9 thresholds is shown in Fig. 11, with different colors representing clusters of tasks known to be related to the same collecting event (same or very similar label information), and with edge thickness representing the distance strength between tasks. Since the tasks change their position in the graphs due to adaptation in best displaying formed clusters, the colors are used to track changes in cluster formation when varying the threshold, without having to rely on the node labels. While a threshold of 0.9 leads to all correctly detected clusters, the requirement of high similarity among tasks leads to non-inclusion of tasks into a cluster (all colored clusters are missing nodes on the right graph) or to splits of a cluster (the blue, cyan and green clusters on the right graph). On the other hand, a more flexible threshold of 0.8 leads to incorrectly detected clusters (the red nodes on the left graph) and splits as certain clusters get larger (the blue cluster on the left graph). For this particular experiment, the 0.85 threshold (center graph) gives a good balance (the blue cluster is almost fully detected, yet the cyan cluster has one task misplaced in another cluster). Despite the difficulty in establishing a good threshold, the results of this clustering facilitates improving the quality of transcriptions, especially given that $32 \%$ of the 
tasks were detected as belonging to a cluster. Manual inspection of all the clusters indicate that presenting the cluster as an additional crowdsourcing task for workers to choose the best value for each field in a cluster, can lead to improvements in all of the cases in this experiment. In some extreme cases, it is possible to assign collection dates to tasks without this information on the label, provide complete or correct scientific author, remove single letter typos that are allowed by lossy algorithms, and have complete descriptions of location and habitat.

Table 5. Clusters of Tasks Reaching Consensus

\begin{tabular}{lcccc}
\hline Score Threshold & \# of Tasks Evaluated & \# Clusters Formed & \# Tasks Clustered & \# Edges \\
\hline 0.80 & 300 & 39 & 117 & 182 \\
0.85 & 300 & 33 & 98 & 147 \\
0.90 & 300 & 27 & 72 & 73 \\
1.0 & 300 & 0 & 0 & 0 \\
\hline
\end{tabular}
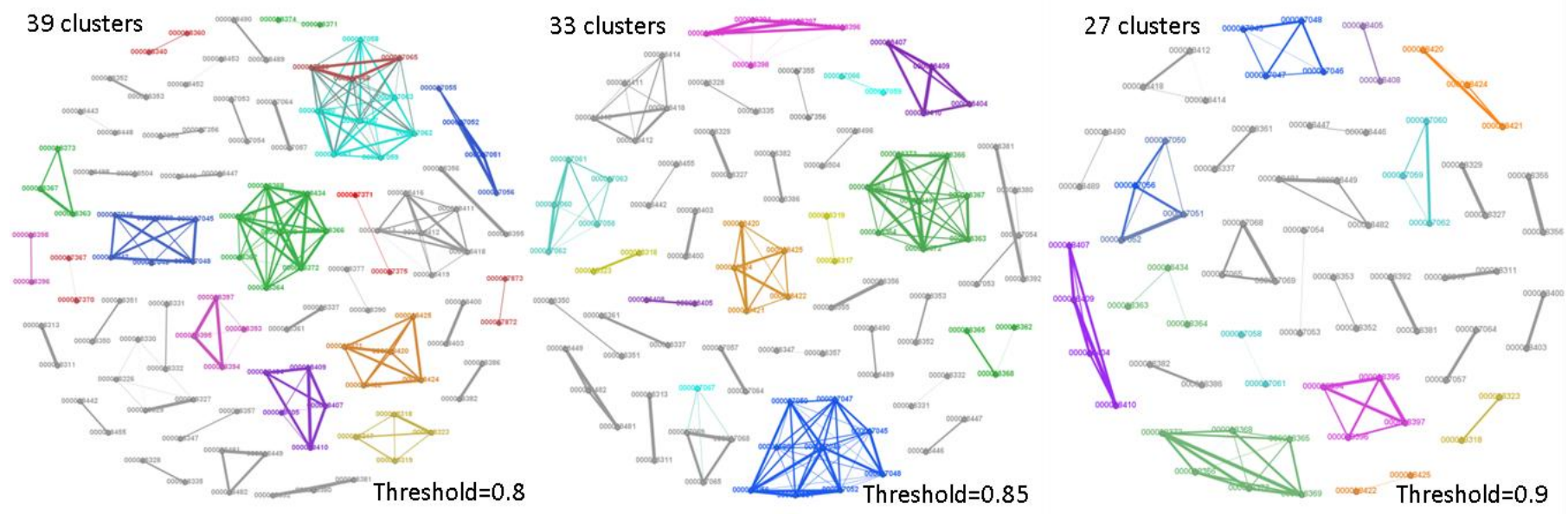

Fig. 11. Clustering of transcription tasks based on consensus result. Increasing threshold for distance between tasks from 0.8 to 0.9 (left to right), different number and copmposition of clusters are found. Nodes represent transcription tasks and tasks change their position in the graph according to the cluster formation. Across all graphs, distinct colors represent tasksthat are known to belong to the same cluster, and red nodes on the 0.8-threshold graph indicate incorrect cluster formation. The blue cluster is partitioned into two clusters with 0.8 and 0.9 -thresholds, and fully clustered with 0.85 -threshold.

\section{Lessons Learned and Future Work}

Several strategies emerged from our exploration of crowdsourced data - some are general in nature and could be reused in other contexts while others were very specific to the data at hand. In this section we focus on the cases that could lead to further improvements. These are separated into (a) improvements to the design of crowdsourcing tasks that impact the crowdsourcing platform, (b) improvements to the consensus algorithms and worker controller, and (c) improvements beyond existing crowdsourcing tools.

\subsection{Task Design Improvement recommendations}

Our results demonstrate the significant impact of the user interface design on the transcriptions produced. The more restricted the user interface for user input (e.g., through the use of drop-down lists and fine-grained fields), the fewer workers the algorithms required to reach consensus. However, caution needs to be applied in order to not constrain the user too much. For example, in the dataset evaluated, collection date had only fully filled dates with day, month and year. Some labels, however, display only month and year. All cases with partial dates resulted in blank/unknown responses in the NotesFromNature output, though users could have accurately specified partial information. This interface also did not allow a range of dates to be entered, in which case different users could have chosen to select either the start or the end date in the range. At the other end of this spectrum, location and habitat are broadly defined fields that could be engineered to capture more parsed data. For example, a normalizer for georeferences and location specifications based on Public Land Survey System (PLSS) would not be necessary if specific fields for this information were offered. With the catch-all text field, this information was entered with variations in encoding (e.g., 'deg', 'o', '*', and 'o' were used by different workers to indicate the degrees portion in latitude and longitude), and variations in spacing, punctuation and ordering (e.g., section/township/range vs. township, range, section). Normalizing this information is important to facilitate the use of the data for biological research that depend on geography. 
As observed in the data produced by the expert and captured by the clustering algorithm, some missing information can be recovered by observing the relationship among multiple specimens. In particular, a set of related specimens often receive the same number from the collector. For this particular scenario, we recommend the creation of a more complex workflow, where first the volunteer is asked to enter the collector number and collection date. Based on this information, and existence of previously crowdsourced data, the user can be given the option to fill the rest of the form with existing data. If the consensus of this task is that multiple users agree with the imported information, both tasks can be updated to have matching information.

Training the transcriber on the idiosyncrasies of specimen labels is likely to increase the accuracy of the output. This could be accomplished by offering help in the interface, forcing users to go through a task catered to show common mistakes before any real work can be performed, or triggering training when low scores are detected for a user. In this experiment, this factor was observed to be most pronounced with scientific name and author, with some users failing to separate authors from the name. Clarifications on which names would be preferred in the presence of multiple scientific names would also lead to less variation in the responses.

\subsection{Additional Improvements to Consensus Algorithms}

The code for the proposed work was developed in Python and is open source [26]. The code is modular to allow custom configurations with algorithms selected on a per-field basis and to allow it to grow with external contributions. For example, we did not make use of dictionaries for correction of misspellings because experiments showed a high rate of error in detecting these cases, due to the high use of abbreviations, technical terms, and proper names. Two future improvements are to select good dictionaries for specific fields, and to detect misspellings using both the dictionary and the transcriptions of other workers. We believe that domain scientists should provide advice on authoritative dictionaries specific to their collections (e.g., specific to their taxon or locality). We could also re-use scientific name parsers developed by the collections community to improve normalization of scientific names and authorship. This was avoided in this work due to the high number of exceptions present in the nomenclatural codes.

Our consensus algorithm selects the best entry within a group of similar responses when using lossy algorithms. Therefore, an algorithm that would merge entries in the same group is also left as future work, since it might require NLP algorithms to ensure that the merged version has not changed semantics of the information and is still accurate. The production of "pretty" outputs is closely related to merging multiple responses. These would contain correct capitalization of words, normalized punctuation, the best encoding alternative (when using special characters outside of the ASCII range), and removal of redundant information. This is expected to improve minimally the accuracy of responses (to match those from the expert), but would produce an output that is more friendly to downstream users of the data.

Even though minimal, there are inaccuracies in the consensus data. To improve the reliability of our solution, cross-referencing information from different fields is also desirable. For example, we could ensure that counties are known to be part of the corresponding states and countries, and that the georeference is also consistent with this information. Other methods for error detection are discussed by [25], which can be reused to flag cases to be proofed by an expert.

\subsection{Recommendations Beyond Crowdsourcing}

Symbiota [5] and ScioTR [6] are examples of systems taking advantage of OCR and NLP to speed up the crowdsourcing effort. However, it is not yet fully agreed that OCR and NLP produce real benefits given the quality of the output when dealing with certain types of challenging labels (with handwriting, mix of fonts and irregular location as shown in Fig. 1), i.e., it is not clear that the effort to correct OCR output is less than typing the entire content and if workers will have good performance detecting minor errors. Thus, future work will explore the influence of such tools on the response accuracy and minimization of work, in an accountable manner. One of the challenges faced by OCR is image noise (e.g., the specimen itself). At the same time, the first step of the crowdsourcing task in some tools, such as NotesFromNature, is to select the region of the image containing the text to be transcribed. This information can be used to remove the main source of noise from the images. With a more accurate OCR output, this information can be used to weight responses from different users, which could break ties present in strict majority voting.

OCR output, even with many imperfections, could also be leveraged to steer volunteers to tasks that would match their skills. For example, state and county fields become challenging for most volunteers when the location is outside the US. This particular dataset showed that workers could not identify correctly these fields for Japan since it takes a person knowledgeable in Japan's geography to detect the suffixes such as 'gun' as indicators of county. As a consequence regions were entered as state, and cities were entered as state. OCR output could be used to allow the volunteer to select tasks that would take advantage of their skills to produce better responses. Preliminary work during the iDigBio [27] CITSCribe Hackathon to enable public participation in transcription showed that it is possible to cluster words from OCR for this purpose. A consequence of clustering is that the same worker can process very similar tasks in sequence (perhaps simultaneously), which would lead to additional reduction in worker's time to process a task. Fig. 12 shows how such an interface could be implemented using Carrot ${ }^{2}$ and Solr on OCR output even with errors. "LI LI" are barcodes interpreted as text, and the block on the right shows some other OCR imperfections, yet the foam visualization on the left brings different classes of labels: from specific geographic regions (e.g., Alaska and Lake Superior), collectors (e.g., Stephen S. Talbot and H. A. Sierk), and taxonomy (e.g., Lichens, Cryptogams and Acarospora). 
NLP can be applied in two distinct contexts: to parse data from OCR or to parse crowdsourcing data. Both can use NLP to break down information to more specific fields: from the OCR to feed into the crowdsourcing task with the expectation that it will speed up the transcriptions, and from a catch-all field, such as location, into specific fields for georeference and PLSS. The differences will lie on how to deal with potential errors, since machine-created errors can be systematic, whereas the variation created by humans can be very diverse.

In the reverse direction of improving the performance of OCR and NLP through the use of transcriptions from the crowd as training data, we expect to be able to handle a larger number of fonts in OCR and be able to parse new types of data without training sets having to be created by experts for NLP.

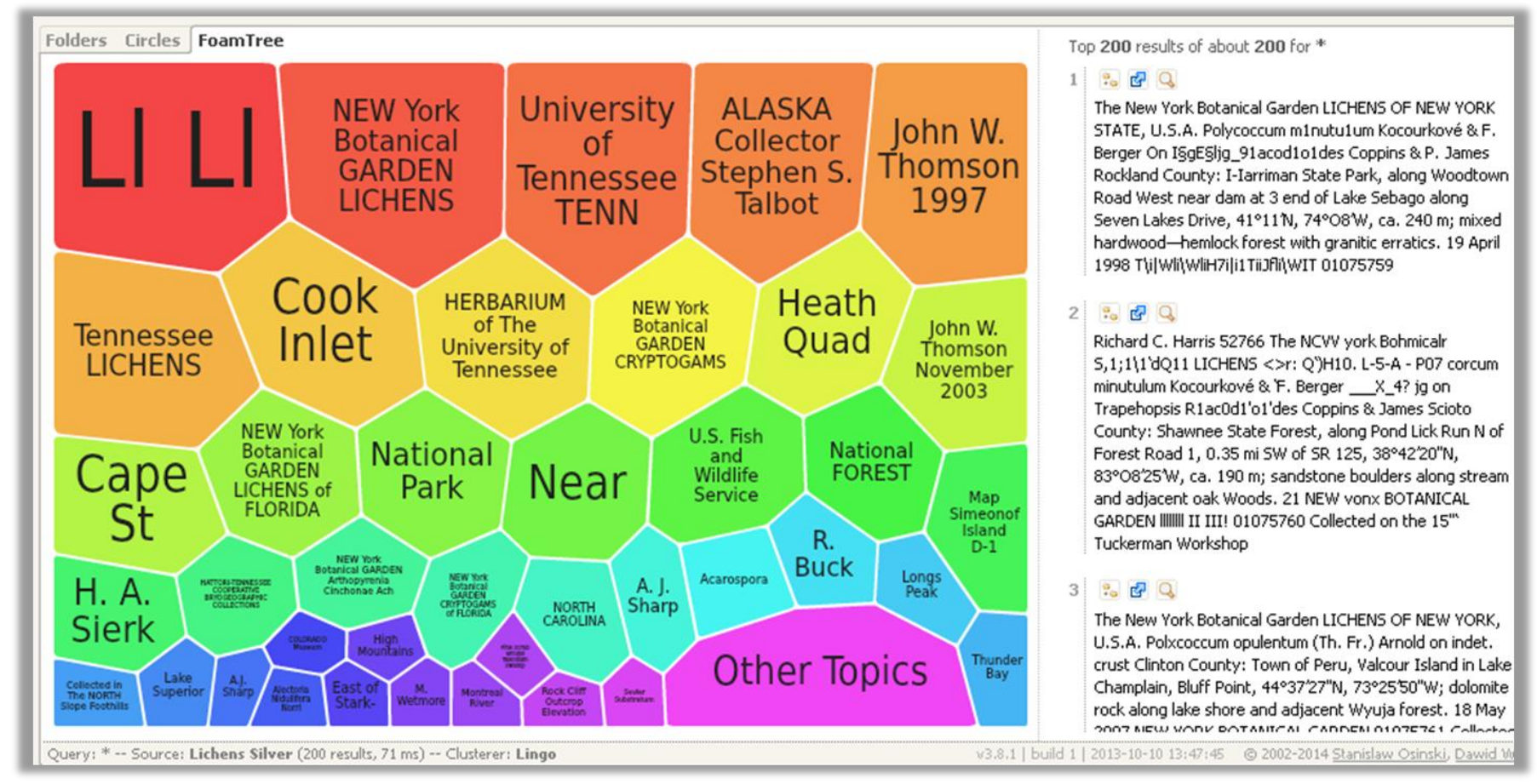

Fig. 12. Clustering of data from Optical Character Recognition (OCR) output (on the right) using Carrot ${ }^{2}$ and Solr can help guide crowdsourcing workers with the selection of transcriptions to perform.

\section{CONCLUSIONS}

To deal with challenges in evaluating textual crowdsourced responses from multiple volunteers, we proposed text-based algorithms to normalize the data and allow approximate comparison of responses. This solution led to a high rate of consensus achievement (a 25 -fold improvement), and the construction of a single answer for the requestor. While some algorithms are domain specific, others will generalize to scenarios requiring textual data comparison. Changes in the voting scheme and similarity comparison algorithms were shown to play a considerable role in consensus for this particular type of task. In addition, we were able to take advantage of the consensus algorithm to dynamically minimize the number of workers required for a given task and for a given field, consequently displaying $37 \%$ and $50 \%$ cost savings respectively when compared to a strategy with fixed number of workers and requiring consensus to be achieved. Additional evaluation of workers and transcriptions were shown to be important in detecting transcription issues generated by workers and by the system at early stages. Clustering algorithms were used to detect similar tasks with good accuracy, potentially leading to improvements in quality and further minimization of work for transcriptions belonging to clusters. We also offered insights to this process towards future improvements and lessons learned.

\section{Acknowledgements}

This work is supported in part by the National Science Foundation (NSF) grant No. EF-1115210, and the AT\&T Foundation. Any opinions, findings and conclusions or recommendations expressed in this material are those of the authors and do not necessarily reflect the views of the NSF or the AT\&T Foundation. 
The authors thank NotesFromNature, Florida State University's Robert K. Godfrey Herbarium, Valdosta State University Herbarium (J. Richard Carter) and University of South Alabama (Kelly M. Major) for providing the data on which this study is based and Joshua Campbell for his contributions to the base code. We gratefully acknowledge the time and energy contributed by the NotesFromNature volunteer community, and the larger community actively engaged in iDigBio working groups with valuable feedback, including participants in the CITSCribe Hackathon. We also acknowledge iDigBio team members for contributing to the context of this work: Larry Page, Pam Soltis, Greg Riccardi, Bruce MacFadden, David Jennings, Joanna McCaffrey, Renato Figueiredo, Gil Nelson, Deb Paul, Elizabeth Ellwood, Alex Thompson, Greg Traub, Dan Stoner, Matt Collins, and Kevin Love.

\section{References}

[1] NSF, Advancing Digitization of Biodiversity Collections (ADBC). [Online]. http://www.nsf.gov/pubs/2013/nsf13569/nsf13569.htm.

[2] E. R. Ellwood, B. A. Dunckel, P. Flemons, R. Guralnick, G. Nelson, G. Newman et al., "Accelerating the Digitization of Biodiversity Research Specimens through Online Public Participation,” BioScience, vol. 65, no. 4, pp. 383-396, April 1, 2015, 2015. DOI:10.1093/biosci/biv005

[3] Zooniverse. NotesFromNature. [Online]. http://notesfromnature.org/.

[4] Atlas of Living Australia, Biodiversity Volunteer Portal. [Online]. http://volunteer.ala.org.au/.

[5] Crowdourcing within Symbiota. [Online]. http://symbiota.org/tiki/tiki-index.php?page=Crowdsourcing.

[6] Micro Support Center, Inc., ScioTR. [Online]. http://www.sciotr.com/.

[7] University College London, Transcribe Bentham. [Online]. http://blogs.ucl.ac.uk/transcribe-bentham/.

[8] T. Causer, J. Tonra, and V. Wallace, "Transcription maximized; expense minimized? crowdsourcing and editing The Collected Works of Jeremy Bentham," Literary and Linguistic Computing, pp. 19, March 28, 2012, 2012. DOI:10.1093/llc/fqs004

[9] Amazon Mechanical Turk. [Online]. https://www.mturk.com/.

[10] PyBossa. [Online]. http://pybossa.com/.

[11] A. Kittur, E. H. Chi, and B. Suh, "Crowdsourcing user studies with Mechanical Turk," in Proceedings of the SIGCHI Conference on Human Factors in Computing Systems, Florence, Italy, 2008, pp. 453-456. DOI:10.1145/1357054.1357127

[12] G. Kazai, J. Kamps, and N. Milic-Frayling, “An analysis of human factors and label accuracy in crowdsourcing relevance judgments,” Inf. Retr., vol. 16, no. 2, pp. 138-178, 2013. DOI:10.1007/s10791-012-9205-0

[13] P. G. Ipeirotis, F. Provost, and J. Wang, "Quality management on Amazon Mechanical Turk," in Proceedings of the ACM SIGKDD Workshop on Human Computation, Washington DC, 2011, pp. 64-67. DOI:10.1145/1837885.1837906

[14] C. Callison-Burch, "Fast, cheap, and creative: evaluating translation quality using Amazon's Mechanical Turk," in Proceedings of the 2009 Conference on Empirical Methods in Natural Language Processing, Singapore, 2009, pp. 286-295.

[15] Q. Gao, and S. Vogel, "Consensus versus expertise: a case study of word alignment with Mechanical Turk," in Proceedings of the NAACL HLT 2010 Workshop on Creating Speech and Language Data with Amazon's Mechanical Turk, Los Angeles, California, 2010, pp. 30-34.

[16] Enki library. [Online] https://github.com/PyBossa/enki/.

[17] S. Nowak, and S. Rüger, "How reliable are annotations via crowdsourcing: a study about inter-annotator agreement for multi-label image annotation," in Proceedings of the international conference on Multimedia information retrieval, Philadelphia, Pennsylvania, USA, 2010, pp. 557-566. DOI: $10.1145 / 1743384.1743478$

[18] H. J. Jung, and M. Lease, "Improving Consensus Accuracy via Z-Score and Weighted Voting," in 2011 AAAI Workshop on Human Computation 2011, pp. $88-90$.

[19] C. Lee, and J. Glass, “A Transcription Task for Crowdsourcing with Automatic Quality Control," in 12th Annual Conference of the International Speech Communication Association, Florence, Italy, 2011, pp. 3041-3044.

[20] W. Tang, and M. Lease, "Semi-supervised consensus labeling for crowdsourcing," in SIGIR 2011 Workshop on Crowdsourcing for Information Retrieval (CIR), 2011

[21] A. S. I. D. Lang, and J. Rio-Ross, "Using Amazon Mechanical Turk to Transcribe Historical Handwritten Documents," code $\{4\}$ lib, no. $15,2011$.

[22] G. Little, L. B. Chilton, M. Goldman, and R. C. Miller, "TurKit: human computation algorithms on mechanical turk," in Proceedings of the 23nd annual ACM symposium on User interface software and technology, New York, New York, USA, 2010, pp. 57-66. DOI:10.1145/1866029.1866040

[23] D. R. Karger, O. Sewoong, and D. Shah, "Budget-optimal crowdsourcing using low-rank matrix approximations," in 49th Annual Allerton Conference on Communication, Control, and Computing (Allerton) 2011 Monticello, IL, 2011, pp. 284-291. DOI:10.1109/Allerton.2011.6120180

[24] V. I. Levenshtein, "Binary codes capable of correcting deletions, insertions and reversals," in Soviet physics doklady, 1966, pp. 707-710.

[25] A. D. Chapman, "Principles and Methods of Data Cleaning - Primary Species and Species-Occurrence Data, version 1.0". Report for the Global Biodiversity Information Facility, Copenhagen. 2005.

[26] Crowd consensus tool. [Online]. Available: http://github.com/idigbio-citsci-hackathon/CrowdConsensus/.

[27] Integrated Digitized Biocollections (iDigBio). [Online]. Available: http://idigbio.org/ 


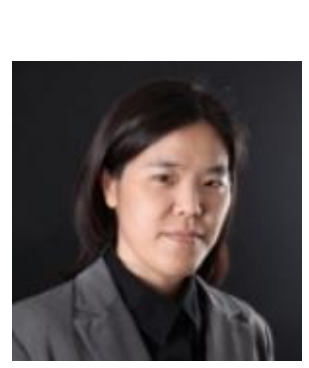

Vitae

Andréa Matsunaga received her $\mathrm{PhD}$ degree in Electrical and Computer Engineering from the University of Florida in 2010. She joined the faculty of the Department of Electrical and Computer Engineering of the University of Florida as an Assistant Research Professor in 2011. Her research interests are in the areas of cloud/grid/distributed computing, information management, machine learning, autonomic computing, and computer architecture.

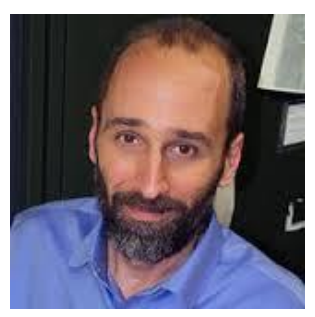

Austin Mast is an Associate Professor in Biological Science at Florida State University, where he also serves as Director of FSU's Robert K. Godfrey Herbarium and Associate Director of FSU's Institute for Digital Information and Scientific Communication. His research focuses on the interplay of ecology and evolution that led to Earth's biodiversity and the generation of digital information about biodiversity specimens that brings that interplay into sharper focus. He received his Ph.D. in Botany from University of Wisconsin-Madison in 2000 and served as a Postdoctoral Scholar at the University of Zurich's Institute for Systematic Botany from 2000-3.

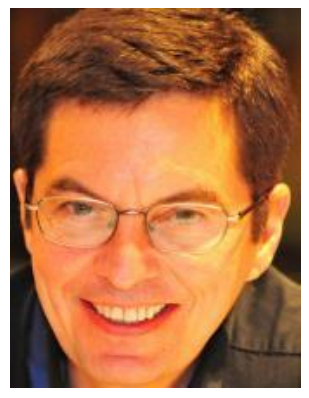

José A. B. Fortes was in the faculty of the School of Electrical Engineering of Purdue University at West Lafayette, Indiana, from 1984 to 2001. In 2001, he joined both the Department of Electrical and Computer Engineering and the Department of Computer and Information Science and Engineering of the University of Florida as Professor and BellSouth Eminent Scholar. His research interests are in the areas of distributed computing, autonomic computing, computer architecture, parallel processing, and fault-tolerant computing. José Fortes is a fellow of the Institute of Electrical and Electronics Engineers (IEEE) professional society. He was a Distinguished Visitor of the IEEE Computer Society from 1991 to 1995. 